\title{
A Case of Post-Streptococcal Glomerulonephritis with Diffuse Alveolar Hemorrhage
}

\begin{abstract}
Acute post-streptococcal glomerulonephritis (PSGN) is characterized by an abrupt onset of edema, hypertension, and hematuria. Life-threatening diffuse alveolar hemorrhage (DAH) is rarely associated with acute PSGN. There have been only two reported cases worldwide, and no case has been reported previously in Korea. Here, we present a patient who clinically presented with pulmonary-renal syndrome; the renal histology revealed post-infectious glomerulonephritis of immune complex origin. A 59-yr-old woman was admitted with oliguria and hemoptysis two weeks after pharyngitis. Renal insufficiency rapidly progressed, and respiratory distress developed. Chest radiography showed acute progressive bilateral pulmonary infiltrates. The clinical presentation suggested DAH with PSGN. Three days after treatment with high-dose steroids, the respiratory distress and pulmonary infiltrates resolved. Electron microscopy of a renal biopsy specimen sample revealed diffuse proliferative glomerulonephritis with characteristic subendothelial deposits of immune complex ("hump"). The renal function of the patient was restored, and the serum creatinine level was normalized after treatment.
\end{abstract}

Key Words : Post-Streptococcal Glomerulonephritis; Diffuse Alveolar Hemorrhage

\author{
Hye-Young Sung, Chang Hoon Lim, \\ Mi-Jung Shin, Byung-Soo Kim, \\ Young Ok Kim, Ho-Chul Song, \\ Suk Young Kim, Euy Jin Choi, \\ Yoon Sik Chang, Byung Kee Bang
}

Division of Nephrology, Department of Internal Medicine, The Catholic University of Korea, Seoul, Korea

Received : 3 May 2006

Accepted : 13 December 2006

Address for correspondence

Byung Soo Kim, M.D.

Division of Nephrology, Department of Internal

Medicine, St. Paul Hospital, The Catholic University of Korea, 620-56 Junnong-dong, Dongdaemoon-gu,

Seoul 130-709, Korea

Tel : +82.2-958-2491, Fax : +82.2-968-7250

E-mail : kbsnep@yahoo.co.kr

\section{INTRODUCTION}

Occasionally, diffuse alveolar hemorrhage (DAH) is accompanied by acute nephritis. When present, it has been shown to be associated with a systemic autoimmune disease such as Goodpasture's syndrome, systemic lupus erythematosus (SLE), and antineutrophil cytoplasmic antibodies (ANCA)-associated vasculitis or microscopic polyangiitis (1-6). Less frequently, it has been reported to be associated with Henoch-schonlein purpura (7), IgA nephropathy (8) or idiopathic immune complex-associated glomerulonephritis (9). DAH associated with acute post-streptococcal glomerulonephritis (PSGN) is extremely rare; there have been no cases reported in Korea to date. A review of the literature has identified two cases worldwide $(10,11)$. In this report, we describe a patient who clinically presented with pulmonary-renal syndrome; the renal histology revealed post-infectious glomerulonephritis of immune complex origin.

\section{CASE REPORT}

A 59-yr-old woman, previously in good health, was admitted because of generalized edema, progressive oliguria, and blood-tinged sputum. Two weeks prior to admission, the patient developed a sore throat and tonsillar swelling.
On admission, the patient had facial swelling and generalized edema, with a blood pressure of 220/130 $\mathrm{mmHg}$, a regular heart beat of 90 beats $/ \mathrm{min}$, a rapid breathing rate of $25 / \mathrm{min}$, and a temperature of $37.2^{\circ} \mathrm{C}$. On oral examination, left tonsillar swelling and throat injection were observed. Chest auscultation revealed coarse breath sounds with rales at both lower lung fields. Chest radiography showed bilateral diffuse pulmonary infiltrates with blunting of both costophrenic angles (Fig. 1A). Respiratory tract infection was suspected, and blood culture and sputum examination were performed. Intravenous antibiotic treatment was started with a third-generation cephalosporin.

Laboratory studies revealed an elevated white blood cell count $(22,000 / \mu \mathrm{L})$ (segmented neutrophil $83 \%$, lymphocyte $12.3 \%$, eosinophil $0.1 \%$, basophil $0.2 \%$ ). The hemoglobin level was normal $(13.4 \mathrm{~g} / \mathrm{dL})$, and the platelet count was normal $\left(228 \times 10^{9} / \mathrm{L}\right)$. C-reactive protein and erythrocyte sedimentation rate were slightly elevated $(11.2 \mathrm{mg} / \mathrm{L}$ and $74 \mathrm{~mm} /$ $\mathrm{hr}$, respectively). The plasma creatinine was $2.3 \mathrm{mg} / \mathrm{dL}$, and the urea was $52.3 \mathrm{mg} / \mathrm{dL}$. The concentrations of aspartate aminotransferase (AST), alanine aminotransferase (ALT), total bilirubin, direct bilirubin, alkaline phosphatase, and creatine kinase (CK) were all normal ( $40 \mathrm{U} / \mathrm{L}, 45 \mathrm{U} / \mathrm{L}, 1.0 \mathrm{mg} / \mathrm{dL}, 0.3$ $\mathrm{mg} / \mathrm{dL}, 338 \mathrm{IU} / \mathrm{L}$, and $147 \mathrm{IU} / \mathrm{L}$, respectively). Lactate acid dehydrogenase (LDH) was elevated to 1,103 IU/L. Urinalysis showed a large number of dysmorphic red blood cells and sev- 
eral WBCs on microscopic examination. The urine chemistry showed proteinuria at $1.2 \mathrm{~g} / 24 \mathrm{hr}$ at Serum C3 was $10.5 \mathrm{mg} /$ $\mathrm{dL}$ (normal $90-180 \mathrm{mg} / \mathrm{dL}$ ), CH50 was below $5 \mathrm{U} / \mathrm{mL}$ (nor$\mathrm{mal} 30-45 \mathrm{U} / \mathrm{mL})$ and, $\mathrm{C} 4$ was $19.4 \mathrm{mg} / \mathrm{mL}(10-40 \mathrm{mg} / \mathrm{mL})$. The serum IgG, IgA, IgE, and IgM were all normal $(795 \mathrm{mg} /$ $\mathrm{dL}, 307 \mathrm{mg} / \mathrm{dL}, 105 \mathrm{mg} / \mathrm{dL}$, and $126 \mathrm{mg} / \mathrm{dL}$, respectively). The anti-streptolysin-O titer (ASO) was 275.4 (normal 0-166 IU/ $\mathrm{mL})$. Autoantibodies including antineutrophil cytoplasmic autoantibody (ANCA), cryoglobulin, antinuclear antibody (ANA), anti-dsDNA antibody, and anti-phospholipid antibody were all negative. The indirect immunofluorescence assay for anti-glomerular basement membrane (GBM) IgG and $\operatorname{IgA}$ were negative. The triple antibody assays for Hantaan virus, Leptospira, R.tustusgamushi were negative. The anti-HIV Ab was negative. The HBs Ag and anti-HCV Ab were negative. Anti-HBs Ab was positive. The anti-mycoplasma antibody was negative. Group A $\beta$-hemolytic streptococcus was cultured from the throat. The blood and urine cultures were negative. Sputum examination showed numerous red cells and hemosiderin-laden macrophages. The sputum for acid-fast bacilli was negative. On the third hospital day, the patient developed red brick-colored hemoptysis and respiratory distress; the clinical status deteriorated progressively with moderate respiratory failure. The chest radiography revealed a marked infiltrative haziness or edema over the whole lung field (Fig. 1B). The hemoglobin level decreased to $9.8 \mathrm{~g} / \mathrm{dL}$ and hematocrit to $27.8 \%$ after hemoptysis developed. The arterial blood gas analysis showed a $\mathrm{PH} 7.39, \mathrm{PCO}_{2} 33.7 \mathrm{mmHg}, \mathrm{PO}_{2}$ $58 \mathrm{mmHg}, \mathrm{HCO}_{3} 20.9$, and $\mathrm{SaO}_{2}$ 92.5\%. Anuria developed and generalized edema progressed despite the use of high-dose
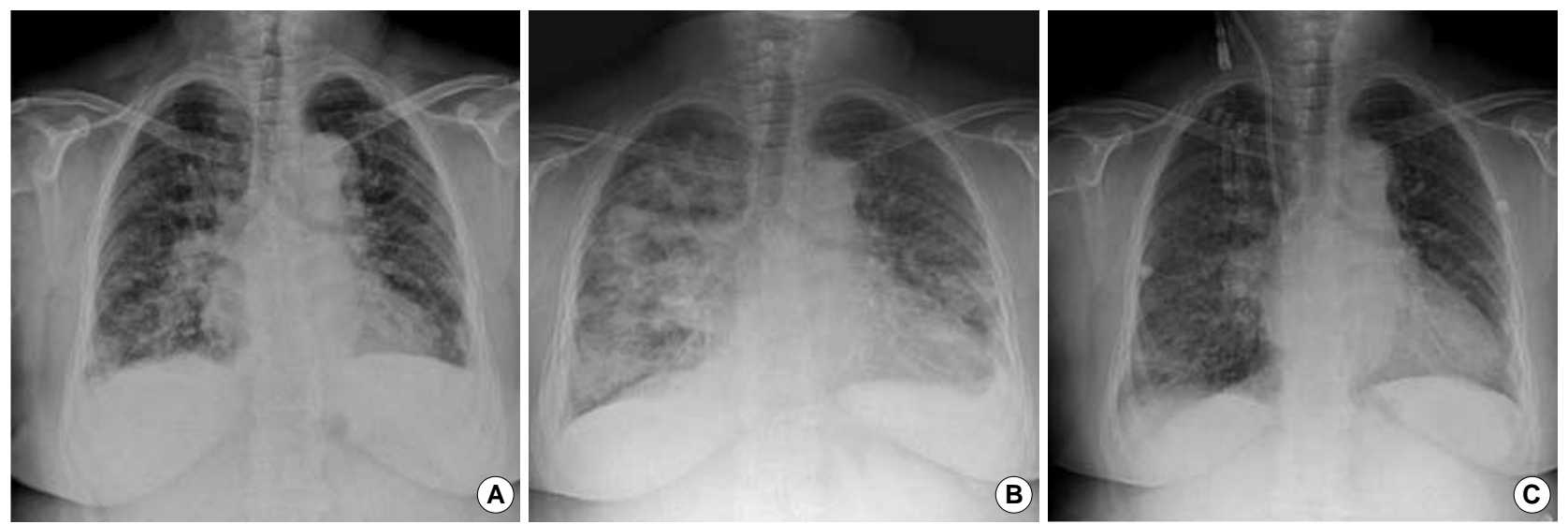

Fig. 1. Chest radiography on admission showed bilateral pulmonary infiltrates with blunting of both costophrenic angles (A), and chest radiography on the fourth hospital day showed that the pulmonary edema and infiltration were progressing $(B)$. The chest abnormalities resolved after three days of steroid treatment $(\mathrm{C})$.
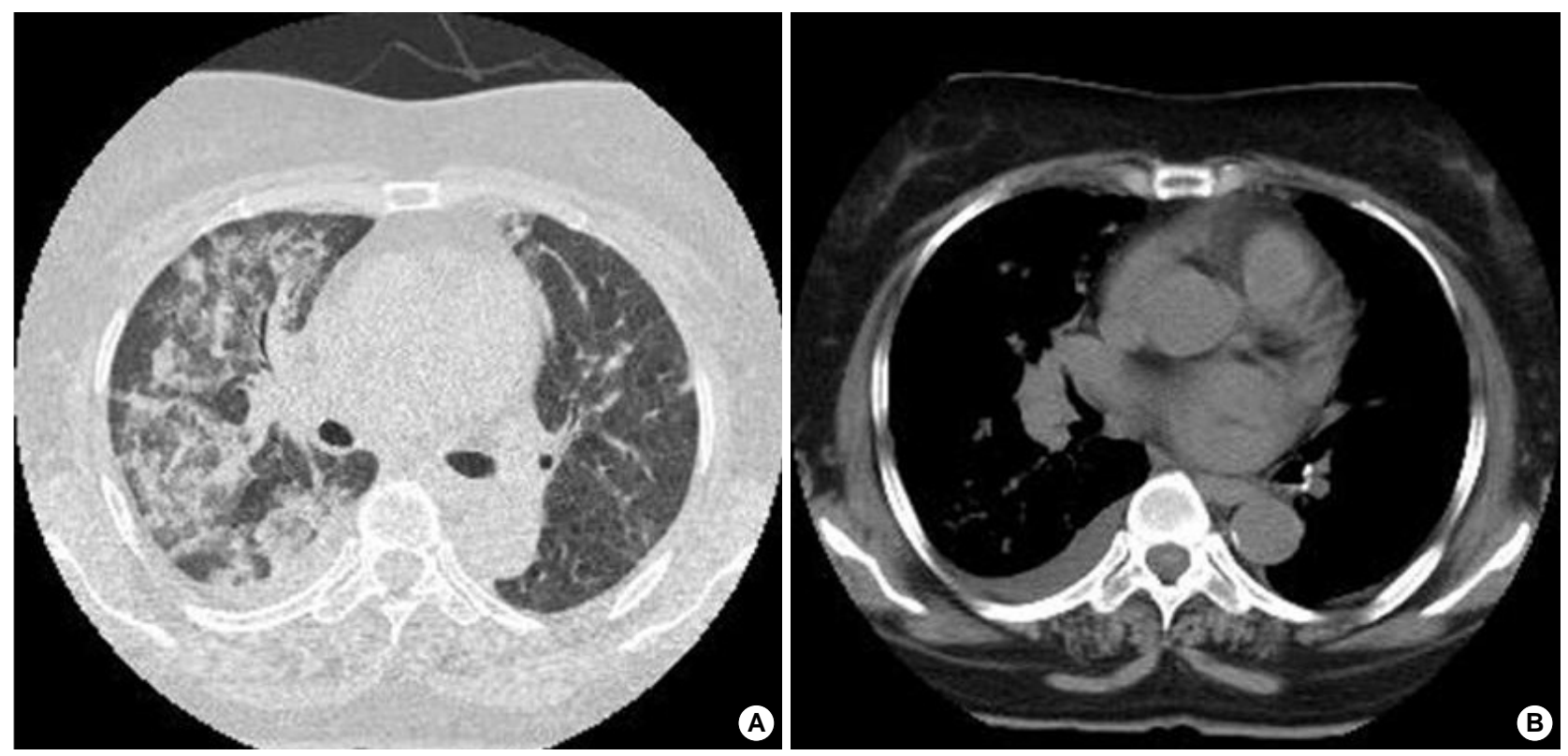

Fig. 2. The CT revealed bilateral pleural effusion and alveolar consolidation or alveolar hemorrhage at both middle and lower lung fields. 
diuretics. The renal function progressively declined, with the creatinine values increasing to $2.8 \mathrm{mg} / \mathrm{dL}$ and the urea to 78.4 $\mathrm{mg} / \mathrm{dL}$. Intermittent hemodialysis was performed. Despite volume control with ultrafiltration, a follow-up chest radio-

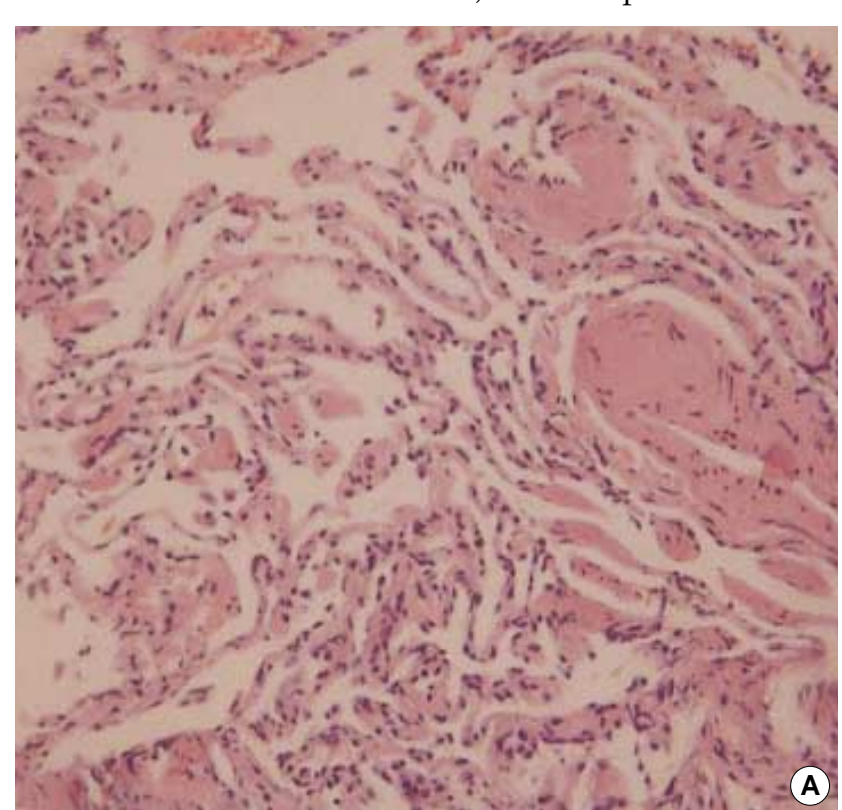

graphy revealed more advanced lung disease. A chest CT was performed (Fig. 2), which revealed bilateral pleural effusion and alveolar consolidation or alveolar hemorrhage in both middle and lower lung fields. Bronchoscopy was performed. Numer-

Fig. 3. The lung biopsy on light microscopy showed eosinophils, neutrophils, and nuclear dust deposition in the alveolar interstitium, septal thickening, and fibrin clots attached to the interalveolar septa (A, H\&E $\times 200 ; B, H \& E \times 400)$.

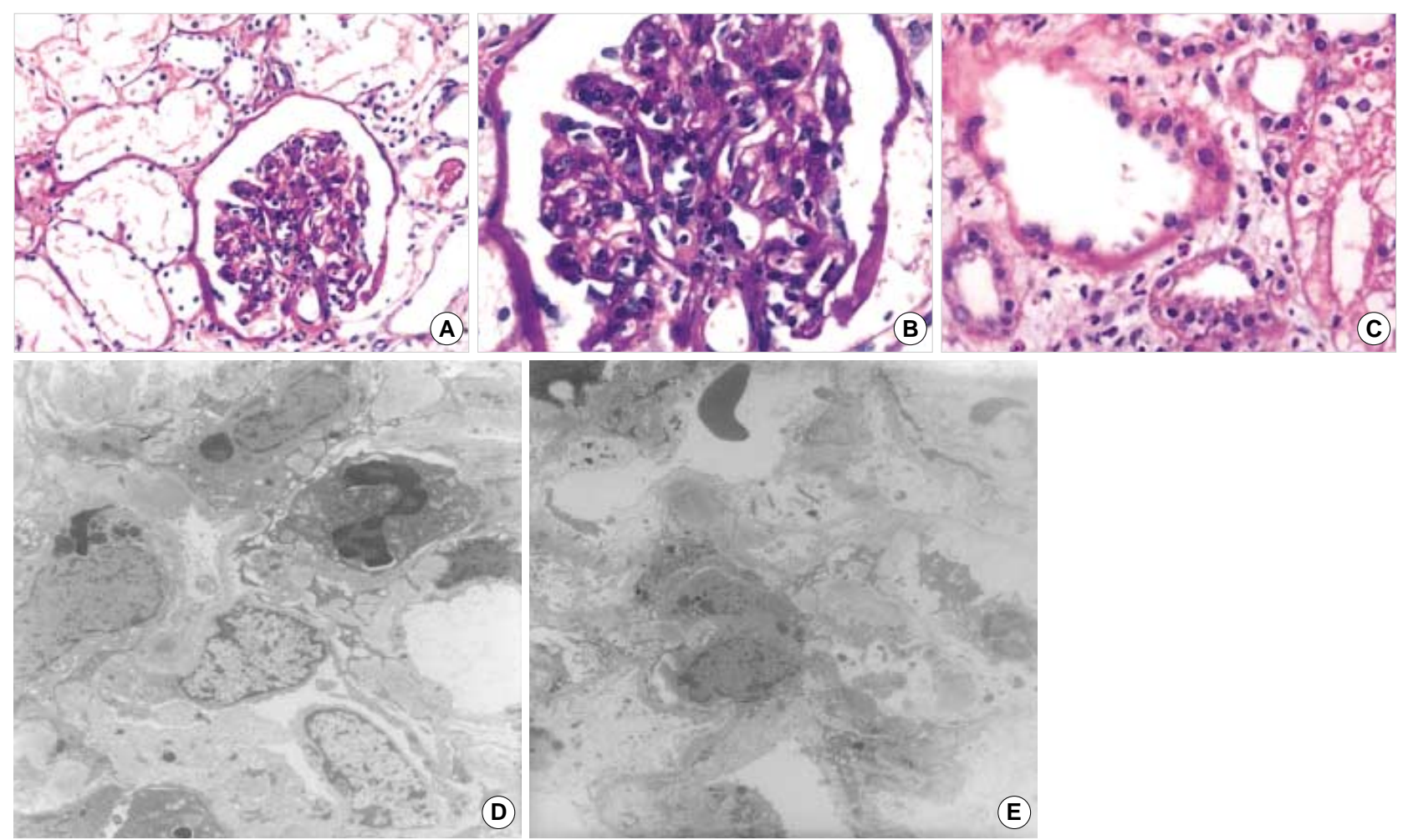

Fig. 4. The renal biopsy specimen on light microscopy showed proliferative glomerulonephritis with neutrophils in the masangium (A, H\&E $\times 200, B, H \& E \times 400 ; C, H \& E \times 400$ ). Electron microscopy showed mesangial hypercellularity with neutrophils and subendothelial electron-dense deposits (D, E). 
ous blood clots were observed in both middle and lower lung fields. Macroscopically hemorrhagic fluid was obtained from a broncho-alveolar lavage. Because of the absence of clinical findings suggestive of typical bacterial pneumonia and the presence of evidence for streptococcal infection such as medical history of upper respiratory infection, physical finding of tonsilar swelling, and laboratory finding of a high ASO titer, we discontinued the ceftriaxone and started daily doses of injected methylprednisolone (1,000 mg) for three days. After three days of steroid treatment the respiratory distress, hemoptysis and edema resolved. The chest abnormalities also resolved (Fig. 1C). The bronchial fluid was negative for bacterial culture, gram stain, or AFB smear. The cell block result from the bronchial washing fluid showed acute inflammatory cells and hemosiderin-laden macrophages. Lung biopsy revealed eosinophils, polymorphonuclear cells and nuclear dust deposition in the alveolar interstitium as well as irregular septal thickening and fibrin clots attached to the interalveolar septa (Fig. 3).

Sixteen days after admission, the renal function recovered. The serum creatinine level and serum ASO titer were normalized $(0.8 \mathrm{mg} / \mathrm{dL}$ and $165 \mathrm{IU} / \mathrm{mL}$, respectively). The patient was discharged without symptoms after 18 days of hospitalization. The patient has been doing well without any additional symptoms; she is currently treated as an outpatient with an ACE inhibitor because of mild proteinuria.

\section{Histological findings of the renal biopsy}

The renal biopsy was performed under ultrasound sonography guidance on the fourth hospital day. Under light microscopy evaluation, the kidney biopsy specimen showed abundant glomeruli, all of which were diffusely and markedly hypercellular with various degrees of infiltration by polymorphonuclear neutrophils and fibrin/platelet aggregates. There were no crescent, vasculitis, or basement membrane duplication interstitium; the tubules and blood vessel were normal (Fig. 4A-C). Under electron microscopy assessment, there were dome-shaped or flame-shaped electron-dense granular subendothelial deposits ("hump”) (Fig. 4D, E).

\section{DISCUSSION}

PSGN is the prototypical post-infectious glomerulonephritis. Glomerulonephritis develops, on average, 10 days after pharyngitis or two weeks after skin infection (impetigo) with a nephritogenic strain of group $A \beta$-hemolytic streptococcus (12). Gross hematuria, oliguric acute renal failure, edema, and hypertension are the classic manifestations; however, most patients present with a milder disease (13). It is a very unusual event for diffuse alveolar hemorrhage (DAH) to develop in association with PSGN. It is not clear why our patient developed such a rare and life-threatening condition, given the fairly typical course of the acute PSGN. DAH is usually associ- ated with Goodpasture's syndrome with crescent glomerulonephritis and pulmonary hemorrhage as the typical characteristic features (1). Goodpasture's syndrome has been shown to have circulating anti-GBM antibody; this antibody is observed as linear deposits on basement membrane $(14,15)$. In our case, there was no circulating anti-GBM antibodies detected. Renal biopsy revealed diffuse proliferative GN without crescent formation on light microscopy. In addition, the granular deposition, of immune complexes on the subendothelium, excluded the possibility of the glomerulonephritis due to anti-GBM disease. DAH has been identified in other causes of immune-mediated nephritis such as SLE, Wegener's granulomatosis, microscopic polyarteritis, mixed cryoglobulinemia, Behcet's syndrome, Henoch-schonlein purpura, and pauch-immune glomerulonephritis $(3-7,16,17)$. In our case, lupus nephritis and cryoglobulinemia were excluded because of the absence of characteristic clinical features as well as negative anti-nuclear antibodies, anti-dsDNA Ab, and cryoglobulins. In addition, the absence of histological evidence of vasculitis in renal tissue also excluded the possibility of systemic vasculitis such as microscopic polyarteritis or ANCA-associated vasculitis. DAH has been reported to be associated with anti-phospholipid antibody syndrome on rare occasions (18); this was excluded because of the absence of anti-phospholipid antibody. On the basis of clinical history, serologic tests, and the pathologic findings of the renal biopsy, the most reasonable diagnosis was PSGN with DAH.

The pathogenic mechanism to explain the presence of DAH with PSGN is not well established. There are some reports that suggest that the clinical picture of Goodpasture's syndrome may not always be etiologically related to anti-GBM disease; in some cases an immune complex mechanism may be operative. Fukuda et al. reviewed an autopsy case of DAH with acute nephritis (19). The lungs showed hemorrhagic interstitial pneumonia, with granular patterns of $\mathrm{IgG}$ and $\mathrm{C} 3$ along the alveoli by immunofluorescence and electron-dense subepithelial deposits with electron microscopy. The kidney had crescent and segmental necrotizing glomerulonephritis associated with membranous nephropathy. Electron microscopy showed numerous subepithelial deposits, and immunoelectron microscopy revealed that $\mathrm{IgG}$ was not present in the GBM itself but present in the subepithelial deposits. Anti-GBM antibody activity was not detected in the serum or the kidney evaluated. These findings suggest that the renal and pulmonary lesions occurred by a similar mechanism in association with immune complex deposits (19). Immune complex deposition on broncho-alveolar epithelium and capillary basement membrane may induce an acute inflammatory reaction with a neutrophilmediated disruption of the alveolar bed and capillary basement membrane. These events may lead to capillitis and DAH (20).

$\mathrm{DAH}$ is a life-threatening condition. If it is recognized late, significant morbidity and mortality would be predicted. Previous uncontrolled observations suggest that high-dose intravenous pulse therapy with corticosteroids produces a prompt 
improvement in the nephritis associated DAH (21). Consistent with expectations, the hemoptysis in our patient as well as the respiratory distress resolved quickly after intravenous methylprednisolone treatment. A review of the literature has identified only two previous cases of PSGN with pulmonary hemorrhage. One case was a 12-yr-old girl who developed a near-fatal pulmonary hemorrhage with acute non-crescent PSGN (10); there was dramatic improvement of hemoptysis and chest radiography abnormalities within 48 to $72 \mathrm{hr}$ after intravenous administration of methylprednisolone; the renal function also improved. The other patient was a 38-yrold male who developed pulmonary hemorrhage with acute crescent PSGN. He also demonstrated dramatic improvement of the hemoptysis and chest radiography abnormalities within $72 \mathrm{hr}$ after steroid treatment; but the renal failure progressed to end-stage disease (11). Leatherman et al. reviewed nine patients with diffuse intrapulmonary hemorrhage and glomerulonephritis not due to anti-GBM antibody, which included systemic vasculitis of unspecified type in two patients with seropositive rheumatoid arthritis, idiopathic crescentic glomerulonephritis with negative immunofluorescence in two, Wegener's granulomatosis in two, and one each of polyarteritis nodosa, Henoch-Schonlein purpura, and mixed connective tissue disease. Pulmonary hemorrhage was reported to improve markedly within 24-72 hr in eight of the nine patients; however, there was no effect on renal function in most of these patients (22). These data suggest that early recognition and timely treatment with corticosteroids could be lifesaving in some patients with DAH and glomerulonephritis.

In conclusion, our case demonstrated that 1) post streptococcal noncrescent glomerulonephritis can be associated with pulmonary hemorrhage, 2) the clinical picture of Goodpasture's syndrome may not always be etiologically related to anti-GBM disease, and in some cases an immune complex mechanism may be implicated, and 3) intravenous administration of highdose steroids, although not proven by controlled studies, may be beneficial for the patients with DAH.

\section{REFERENCES}

1. Lombard CM, Colby TV, Elliott CG. Surgical pathology of the lung in anti-basement membrane antibody-associated Goodpasture's syndrome. Hum Pathol 1989; 20: 445-51.

2. Kim YO, Choi JY, Park JI, Yoon SA, Yang CW, Hyoung K, Bang BK. A case of Goodpasture's syndrome with massive pulmonary hemorrhage. J Korean Med Sci 2000; 15: 99-102.

3. Gallagher H, Kwan JT, Jayne DR. Pulmonary renal syndrome: a 4year, single-center experience. Am J Kidney Dis 2002; 39: 42-7.

4. Zamora MR, Warner ML, Tuder R, Schwarz MI. Diffuse alveolar hemorrhage and systemic lupus erythematosus. Clinical presentation, histology, survival, and outcome. Medicine (Baltimore) 1997; 76: 192-202.

5. Niles JL, Bottinger EP, Saurina GR, Kelly KJ, Pan G, Collins AB,
McCluskey RT. The syndrome of lung hemorrhage and nephritis is usually an ANCA-associated condition. Arch Intern Med 1996; 156: 440-5.

6. Travis WD, Colby TV, Lombard C, Carpenter HA. A clinicopathologic study of 34 cases of diffuse pulmonary hemorrhage with lung biopsy confirmation. Am J Surg Pathol 1990; 14: 1112-25.

7. Nadrous HF, Yu AC, Specks U, Ryu JH. Pulmonary involvement in Henoch-Schonlein purpura. Mayo Clin Proc 2004; 79: 1151-7.

8. Fung M, Churchill D, Alexopoulou I, Ingram A. IgA nephropathy and pulmonary hemorrhage in an adult. Am J Nephrol 2001; 21 : 318-22.

9. Loughlin GM, Taussig LM, Murphy SA, Strunk RC, Kohnen PW. Immune-complex-mediated glomerulonephritis and pulmonary hemorrhage simulating Goodpasture syndrome. J Pediatr 1978; 93: 181-4.

10. Gilboa N, McIntire S, Hopp L, Ellis D. Acute noncrescentic poststreptococcal glomerulonephritis presenting with pulmonary hemorrhage. Pediatr Nephrol 1993; 7: 147-50.

11. Chugh KS, Gupta VK, Singhal PC, Sehgal S. Case report: poststreptococcal crescentic glomerulonephritis and pulmonary hemorrhage simulating Goodpasture's syndrome. Ann Allergy 1981; 47: 104-6.

12. Hahn RG, Knox LM, Forman TA. Evaluation of poststreptococcal illness. Am Fam Physician 2005; 71: 1949-54.

13. Sarkissian A, Papazian M, Azatian G, Arikiants N, Babloyan A, Leumann E. An epidemic of acute postinfectious glomerulonephritis in Armenia. Arch Dis Child 1997; 77: 342-4.

14. Salama AD, Levy JB, Lightstone L, Pusey CD. Goodpasture's disease. Lancet 2001; 358: 917-20.

15. Salama AD, Dougan T, Levy JB, Cook HT, Morgan SH, Naudeer S, Maidment G, George AJ, Evans D, Lightstone L, Pusey CD. Goodpasture's disease in the absence of circulating anti-glomerular basement membrane antibodies as detected by standard techniques. Am J Kidney Dis 2002; 39: 1162-7.

16. Jara LJ, Vera-Lastra O, Calleja MC. Pulmonary-renal vasculitic disorders: differential diagnosis and management. Curr Rheumatol Rep 2003; 5: 107-15.

17. Bosch X, Font J. The pulmonary-renal syndrome: a poorly understood clinicopathologic condition. Lupus 1999; 8: 258-62.

18. Crausman RS, Achenbach GA, Pluss WT, O’Brien RF, Jennings CA. Pulmonary capillaritis and alveolar hemorrhage associated with the antiphospholipid antibody syndrome. J Rheumatol 1995; 22: 554-6.

19. Fukuda Y, Yamanaka N, Ishizaki M, Suzuki T, Masugi Y, Yajima G, Nagata T. Immune complex-mediated glomerulonephritis and interstitial pneumonia simulating Goodpasture's syndrome. Acta Pathol Jpn 1982; 32: 361-70.

20. Green RJ, Ruoss SJ, Kraft SA, Duncan SR, Berry GJ, Raffin TA. Pulmonary capillitis and alveolar hemorrhage. Update on diagnosis and management Chest 1996: 110: 1305-16.

21. De Torrente A, Popovtzer MM, Guggenheim SJ, Schrier RW. Serious Pulmonary hemorrhage, glomerulonephritis and massive steroid therapy. Ann Intern Med 1975; 83: 218-9.

22. Leatherman JW, Sibley RK, Davies SF. Diffuse intrapulmonary hemorrhage and glomerulonephritis unrelated to anti-glomerular basement membrane antibody. Am J Med 1982; 72: 401-10. 